\title{
Fakeons and the classicization of quantum gravity: the FLRW metric
}

\author{
Damiano Anselmi \\ Dipartimento di Fisica "Enrico Fermi", Università di Pisa and INFN, Sezione di Pisa, \\ Largo B. Pontecorvo 3, 56127 Pisa, Italy \\ E-mail: damiano.anselmi@unipi.it
}

ABstRACT: Under certain assumptions, it is possible to make sense of higher derivative theories by quantizing the unwanted degrees of freedom as fakeons, which are later projected away. Then the true classical limit is obtained by classicizing the quantum theory. Since quantum field theory is formulated perturbatively, the classicization is also perturbative. After deriving a number of properties in a general setting, we consider the theory of quantum gravity that emerges from the fakeon idea and study its classicization, focusing on the FLRW metric. We point out cases where the fakeon projection can be handled exactly, which include radiation, the vacuum energy density and the combination of the two, and cases where it cannot, which include dust. Generically, the classical limit shares many features with the quantum theory it comes from, including the impossibility to write down complete, "exact" field equations, to the extent that asymptotic series and nonperturbative effects come into play.

KeYwords: Models of Quantum Gravity, Classical Theories of Gravity, Beyond Standard Model, Cosmology of Theories beyond the SM

ArXiv EPrint: 1901.09273 


\section{Contents}

1 Introduction 1

2 Fakeon projection in nonrelativistic mechanics 4

2.1 Fakeon averages 5

2.2 Harmonic oscillator with an external force 6

$\begin{array}{lll}3 & \text { Fakeon projection by asymptotic expansion } & 7\end{array}$

4 The nonperturbative fakeon projection $\quad 9$

$\begin{array}{lll}5 & \text { The classical limit of quantum gravity } & 11\end{array}$

6 The classicization of the FLRW solution 12

$\begin{array}{ll}\text { 6.1 Method of the reduced action } & 12\end{array}$

$\begin{array}{ll}6.2 & \text { The FLRW metric } \\ & 13\end{array}$

$\begin{array}{lll}6.3 \text { Projection } & 14\end{array}$

7 Non-higher derivative approach to the FLRW solution 18

$\begin{array}{llr}8 & \text { Conclusions } & 19\end{array}$

\section{Introduction}

Typically, higher derivative quantum field theories propagate ghosts, if they are formulated in the usual ways. The ghosts are unphysical degrees of freedom that cannot be projected away without violating unitarity. Recently, a new quantization prescription [1,2] has been set forth, to quantize various types of degrees of freedom as "fakeons", i.e. fake particles. The main virtue of the fakeons is that they can be projected away from the physical spectrum consistently with unitarity.

The fakeon prescription can be used to turn the ghosts and possibly some physical particles into fake particles. Its main application is to quantum gravity $[1,3,4]$, since one fakeon $\chi_{\mu \nu}$ of spin two, together with a scalar field $\phi$, is able to make the theory renormalizable while preserving unitarity.

In this paper we investigate some remarkable features of the classical limits of the theories of particles and fakeons. We recall that the fakeon quantization prescription has a truly quantum nature, since it amounts to a nonanalytic operation on the loop diagrams, called average continuation. The average continuation is the arithmetic average of the analytic continuations that circumvent the thresholds associated with the processes that involve fakeons $[2,5]$. 


\begin{tabular}{|c|c|c|c|c|}
\hline interim & quantization & quantum & classicization & classical \\
\hline classical theory & fakeon projection & field theory & classical fakeon projection & limit \\
\hline
\end{tabular}

Figure 1. Quantization/classicization scheme.

The idea originates from a thorough analysis of the cutting equations, which are diagrammatic identities that encode the unitarity relation $S^{\dagger} S=1[6-8]$. The fakeons also allow us to reformulate and actually better understand the Lee-Wick models [9-13]. For a review of these topics, see ref. [14].

The backlash of the fakeon prescription on the classical theory turns out to be nontrivial [14], because the quantization process includes an additional step, as shown in figure 1. The starting local action is just an interim one, being unprojected. The finalized classical action can be obtained only after the quantization, and emerges from the classicization of the quantum theory.

The interim classical action of quantum gravity coupled to matter can be expressed in two ways. The standard way is by means of higher-derivative terms [1]:

$$
S_{\mathrm{QG}}(g, \Phi)=-\frac{1}{2 \kappa^{2}} \int \mathrm{d}^{4} x \sqrt{-g}\left[2 \Lambda_{C}+\zeta R+\alpha\left(R_{\mu \nu} R^{\mu \nu}-\frac{1}{3} R^{2}\right)-\frac{\xi}{6} R^{2}\right]+S_{\mathfrak{m}}(g, \Phi) .
$$

Here $\alpha, \xi, \zeta$ and $\kappa$ are real positive constants. We make no assumption on the sign of the cosmological constant $\Lambda_{C}$. The Planck mass is $M_{\mathrm{Pl}}=1 / \sqrt{G}=\sqrt{8 \pi \zeta} / \kappa$. Moreover, $\Phi$ are the matter fields and $S_{\mathfrak{m}}$ is the action of the matter sector. For example, $S_{\mathfrak{m}}$ can be the action of the standard model, or a standard model extension, as long as it is covariantized and contains all the nonminimal couplings that are compatible with renormalizability.

For simplicity, in this paper we work at $\Lambda_{C}=0$ and view the cosmological constant as a component of dark energy. An equivalent version of the interim classical action (1.1) is obtained by means of extra fields, which allow us to remove the higher derivatives. We find [4]

$$
\mathcal{S}_{\mathrm{QG}}(g, \phi, \chi, \Phi)=S_{\mathrm{H}}(g)+S_{\chi}(g, \chi)+S_{\phi}(\tilde{g}, \phi)+S_{\mathfrak{m}}\left(\tilde{g} \mathrm{e}^{\kappa \phi}, \Phi\right),
$$

where $\tilde{g}_{\mu \nu}=g_{\mu \nu}+2 \chi_{\mu \nu}$ and

$$
\begin{gathered}
S_{\mathrm{H}}(g)=-\frac{\zeta}{2 \kappa^{2}} \int \mathrm{d}^{4} x \sqrt{-g} R, \quad S_{\phi}(g, \phi)=\frac{3 \zeta}{4} \int \mathrm{d}^{4} x \sqrt{-g}\left[\nabla_{\mu} \phi \nabla^{\mu} \phi-\frac{m_{\phi}^{2}}{\kappa^{2}}\left(1-\mathrm{e}^{\kappa \phi}\right)^{2}\right], \\
S_{\chi}(g, \chi)=S_{\mathrm{H}}(\tilde{g})-S_{\mathrm{H}}(g)+\int \mathrm{d}^{4} x\left[-2 \chi_{\mu \nu} \frac{\delta S_{\mathrm{H}}(g)}{\delta g_{\mu \nu}}+\frac{\zeta^{2}}{2 \alpha \kappa^{2}} \sqrt{-g}\left(\chi_{\mu \nu} \chi^{\mu \nu}-\chi^{2}\right)\right]_{g \rightarrow \tilde{g}} .
\end{gathered}
$$

As we see, the theory describes the graviton, a scalar field $\phi$ of squared mass $m_{\phi}^{2}=\zeta / \xi$, a spin-2 fakeon $\chi_{\mu \nu}$ of squared mass $m_{\chi}^{2}=\zeta / \alpha$ and the matter fields.

It is easy to show, from the expression of $S_{\chi}$, that the $\chi_{\mu \nu}$ quadratic action is of the Pauli-Fierz type, but with the wrong overall sign [4]. For this reason $\chi_{\mu \nu}$ must be quantized 
as a fakeon. At present we do not know whether $\phi$ should be quantized as a physical particle or a fakeon. Thus, we have two possibilities: one is the graviton/scalar/fakeon (GSF) theory and the other one is the graviton/fakeon/fakeon (GFF) theory. Throughout this paper, we work with the second option, because we plan to investigate the fakeons in the Friedmann-Lemaitre-Robertson-Walker (FLRW) scenario, which is not sensitive to $\chi_{\mu \nu}$.

We recall that if we quantize every degree of freedom by means of the standard Feynman prescription, the action (1.1) gives the Stelle theory [15-19] (after we drop $S_{\mathfrak{m}}$ ). In that case, no projection is possible and the classicization is trivial. However, the Stelle theory propagates ghosts.

The fakeon projection is inherited from quantum field theory, so it is formulated perturbatively. Its classical limit amounts to take the average of the retarded and advanced potentials [14]. What happens when we try and resum the perturbative expansion of the classicization? Can we grasp the "exact" classical field equations and the fakeon projection at the nonperturbative level? In this paper, we investigate these issues and uncover interesting, and to some extent surprising, properties.

At the quantum level, we are accustomed to build a theory perturbatively, by adding, so to speak, quantum after quantum, or interaction after interaction. We do not expect anything like that to occur in a classical framework. One of the surprises of the theory of quantum gravity built on the fakeon idea is precisely that the classical limit shares many features with the quantum theory it comes from, including the impossibility to write down complete, exact field equations. Unless we have knowledge about the nonperturbative sector of quantum gravity, the projected classical field equations we get are also perturbative. In general, asymptotic series come into play and nonperturbative corrections may have to be included. However, in special cases, the resummation can be handled exactly.

We study these issues in a general setting and then concentrate on the FLRW solution of the classicized theory of quantum gravity. We show that the fakeon projection can be handled exactly in the cases of radiation, the vacuum energy density and the combination of both. Instead, in the case of dust it cannot, so asymptotic series are generated and nonperturbative effects may come into play.

Quantum gravity, as it emerges from the fakeon idea, is in line with high-energy particle physics. In particular, it follows from the same principles that lead to the standard model: unitarity, locality and renormalizability [20]. The scattering amplitudes are defined perturbatively by means of Feynman diagrams, which can be calculated with an effort comparable to the one required by analogous computations in the standard model $[3,4]$.

Several proposals for quantum gravity have appeared in the past decades. We mention string theory [21-24], loop quantum gravity [25-27], holography (the AdS/CFT correspondence) [28-31], lattice gravity [32] and asymptotic safety [33-36]. However, their predictive powers are limited. Some proposals, like string theory, have a huge space of free parameters [37, 38]. Others, like the AdS/CFT correspondence, rely on conjectured dualities. Some, like lattice gravity, asymptotic safety and the AdS/CFT correspondence, do not admit perturbative expansions and deal with strongly coupled quantum field theory. Others, like string theory and loop quantum gravity, involve mathematics that is not well understood. 
Here are some of the reasons why we claim that the solution provided by the fakeons is the right theory of quantum gravity. As far as calculability, predictivity and falsifiability are concerned, the fakeon solution tops the competitors by far. Actually, it may be turn out to be the most predictive theory ever, since it is able to cover a huge range of energies (from the infrared limit up to and beyond the Planck scale) perturbatively and with few independent parameters.

The masses $m_{\phi}$ and $m_{\chi}$ of $\phi$ and $\chi_{\mu \nu}$ might be smaller, or even much smaller, than the Planck mass $M_{\mathrm{Pl}}$. The perturbative expansion, which is formulated in powers of the fakeon/graviton fine structure constants $\alpha_{\phi}=m_{\phi}^{2} / M_{\mathrm{Pl}}^{2}$ and $\alpha_{\chi}=m_{\chi}^{2} / M_{\mathrm{Pl}}^{2}$, makes sense as long as the renormalization group flow keeps these parameters smaller than unity, which likely means somewhere above the Planck scale. At some point, up there, nonperturbative effects start to become important. The theory predicts new physics below the Planck scale $[3,4]$, at energies around $m_{\phi}$ and $m_{\chi}$. At low energies, it reduces to the nonrenormalizable theory made of the Hilbert-Einstein action plus the counterterms turned on by renormalization [39]. Note that the low-energy expansion is independent of the prescription with which the fields are quantized.

It is important to stress that the fakeon idea does not make assumptions about the nature of spacetime at infinitesimally small distances. Instead, the new understanding of spacetime at the microscopic level emerges from the theory itself. It is encoded in the violation of microcausality $[4,14]$ : the concepts of space and time, past, present and future, cause and effect lose meaning at energies larger than the lightest fakeon mass. Our present knowledge of the laws of physics leaves enough room for this prediction to be accurate, both from the theoretical and experimental viewpoints.

Over the years, the concept of causality has been gradually put aside in quantum field theory. The reason is that it is not well understood, which makes it hard to elevate it to the rank of a fundamental principle. A definition that matches the intuitive notion is missing [40] and Bogoliubov's proposal [41], which implies the Lehmann-SymanzikZimmermann one (i.e. that the fields commute at spacelike separated points), is an off-shell condition for the Feynman diagrams and the correlation functions. At the experimental level, the difficulty with causality comes from the fact it is hard to localize particles described by relativistic wave packets that are on shell.

The paper is structured as follows. In section 2 we study the fakeons and the classicization in nonrelativistic mechanics. In section 3 we study the asymptotic expansion of the fakeon projection. In section 4 we analyze the issues that arise at the nonperturbative level. In section 5 we recall the basic aspects of the classicization of quantum gravity. In section 6 we study the FLRW solution. In section 7 we give details on how to proceed in the non-higher-derivative approach (1.2). Section 8 contains the conclusions.

\section{Fakeon projection in nonrelativistic mechanics}

In this section and the next one we study the fakeon projection and its resummation in some models of nonrelativistic mechanics, which provide a simple environment where most 


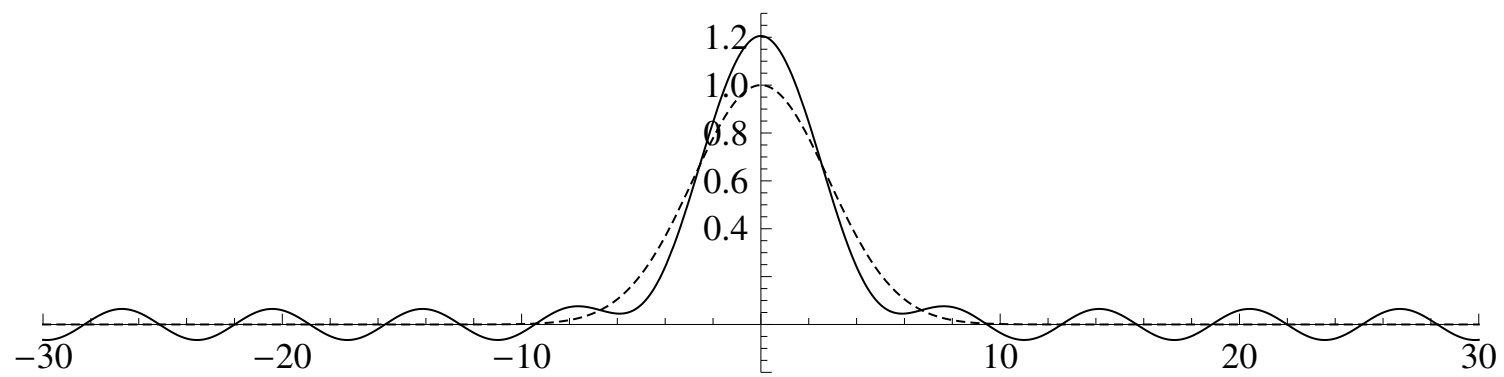

Figure 2. Fakeon average (continuous line) of a Gaussian function (2.6) (dashed line) with $\tau=1$ and $\gamma=1 / 8$.

key conceptual issues are already in play. We consider the higher-derivative Lagrangian

$$
\mathcal{L}_{\mathrm{HD}}=\frac{m}{2}\left(\dot{x}^{2}-\tau^{2} \ddot{x}^{2}\right)-V(x, t),
$$

where $x$ is the coordinate, $m$ is the mass and $\tau$ is a real constant.

The simplest case is $V(x, t)=-x F_{\text {ext }}(t)$, where $F_{\text {ext }}(t)$ is an external force. The unprojected equation of motion is $m K \ddot{x}=F_{\text {ext }}$, where

$$
K=1+\tau^{2} \frac{\mathrm{d}^{2}}{\mathrm{~d} t^{2}}
$$

and the projected one reads

$$
m \ddot{x}=\left\langle F_{\text {ext }}\right\rangle_{K} \cdot
$$

As recalled in the introduction, the classical fakeon average is

$$
\langle A\rangle_{X} \equiv \frac{1}{2}\left[\left.\frac{1}{X}\right|_{\text {rit }}+\left.\frac{1}{X}\right|_{\mathrm{adv}}\right] A,
$$

the subscripts denoting the retarded and advanced potentials, respectively. We find [14]

$$
m \ddot{x}=\int_{-\infty}^{\infty} \mathrm{d} u \frac{\sin (|u| / \tau)}{2 \tau} F_{\text {ext }}(t-u) .
$$

\subsection{Fakeon averages}

Before moving to the cases where the resummation of the fakeon projection plays an important role, it is useful to check out the fakeon average $\left\langle F_{\text {ext }}\right\rangle_{K}$ in some simple examples. If the external force is Gaussian,

$$
F_{\text {ext }}(t)=\exp \left(-\frac{\gamma}{2} t^{2}\right)
$$

the fakeon average returns a wiggling function, as shown in figure 2:

$$
\left\langle F_{\text {ext }}\right\rangle_{K}=\sqrt{\frac{\pi}{2 \gamma}} \mathrm{e}^{-1 /(2 \gamma)} \operatorname{Im}\left[\mathrm{e}^{i t} \operatorname{Erf}\left(\frac{\gamma t+i}{\sqrt{2 \gamma}}\right)\right] .
$$


The average $\left\langle P_{n}(t)\right\rangle_{K}$ of a polynomial $P_{n}(t)$ of degree $n$ is another polynomial $Q_{n}(t)$ of the same degree, which can be determined from $K Q_{n}=P_{n}$. For example, $\langle 1\rangle_{K}=1$, $\langle t\rangle_{K}=t,\left\langle t^{2}\right\rangle_{K}=t^{2}-2 \tau^{2}$. These results can be also verified by taking the limits

$$
\lim _{\gamma \rightarrow 0}\left\langle P_{n}(t) \mathrm{e}^{-\gamma t^{2} / 2}\right\rangle_{K} .
$$

Similarly, we find

$$
\left\langle\mathrm{e}^{i \omega t}\right\rangle_{K}=\frac{\mathrm{e}^{i \omega t}}{1-\omega^{2} \tau^{2}}, \quad\left\langle\mathrm{e}^{i t / \tau}\right\rangle_{K}=\frac{\mathrm{e}^{i t / \tau}}{4 \tau}(\tau-2 i t),
$$

etc., for $\omega<1 / \tau$.

The resummation of the fakeon projection often leads to multiple averages, such as $\left\langle\left\langle F_{\text {ext }}\right\rangle\right\rangle,\left\langle\left\langle\left\langle F_{\text {ext }}\right\rangle\right\rangle\right\rangle$, etc. If we want to know how to handle these expressions, we must go back to the origin of the projection, rooted in quantum field theory. In ref. [2] it was shown that when two or more fakeon thresholds coincide, they must be treated as limits of distinct thresholds. From this property we can easily prove the identity

$$
\lim _{\epsilon \rightarrow 0} \mathcal{P} \prod_{i=1}^{n+1} \frac{1}{x-\epsilon c_{i}}=\frac{(-1)^{n}}{n !} \frac{\mathrm{d}^{n}}{\mathrm{~d} x^{n}} \mathcal{P} \frac{1}{x},
$$

where $\mathcal{P}$ denotes the principal value and $c_{i}$ are arbitrary distinct numbers. This formula allows us to "raise $\mathcal{P}$ to arbitrary powers" and so compute the multiple averages.

Specifically, if $\tilde{F}_{\text {ext }}(\nu)$ is the Fourier transform of $F_{\text {ext }}(t)$, we have

$$
\left\langle F_{\text {ext }}\right\rangle_{K}=\mathcal{P} \int_{-\infty}^{+\infty} \frac{\mathrm{d} \nu}{2 \pi} \frac{\mathrm{e}^{-i \nu t} \tilde{F}_{\text {ext }}(\nu)}{1-\tau^{2} \nu^{2}} .
$$

"Squaring the average" by means of (2.8), we find

$$
\begin{aligned}
\left\langle\left\langle F_{\text {ext }}\right\rangle_{K}\right\rangle_{K} & =\left\langle F_{\text {ext }}\right\rangle_{K}+\frac{1}{2} \frac{\mathrm{d}}{\mathrm{d} t}\left[\left\langle t F_{\text {ext }}\right\rangle_{K}-t\left\langle F_{\text {ext }}\right\rangle_{K}\right] \\
& =\int_{-\infty}^{\infty} \frac{\mathrm{d} u}{4}(\sin |u|-|u| \cos u) F_{\text {ext }}(t-\tau u) .
\end{aligned}
$$

With the help of a limit like (2.7), it is easy to check that $\left\langle\langle 1\rangle_{K}\right\rangle_{K}=1,\left\langle\langle t\rangle_{K}\right\rangle_{K}=t$ and $\left\langle\left\langle t^{2}\right\rangle_{K}\right\rangle_{K}=t^{2}-4 \tau^{2}$. In analogous ways, formulas for more repeated averages can be worked out.

\subsection{Harmonic oscillator with an external force}

The resummation of the projection is important in the next example, which is the harmonic oscillator with an external force:

$$
V(x, t)=\frac{m}{2} \omega^{2} x^{2}-x F_{\text {ext }}(t) .
$$

We view $\omega^{2}$ as the expansion parameter. The unprojected equation of motion is

$$
m K \ddot{x}+m \omega^{2} x=F_{\text {ext }}=m \tilde{K}\left(\frac{\mathrm{d}^{2}}{\mathrm{~d} t^{2}}+\Omega^{2}\right) x,
$$


where

$$
\Omega=\frac{1}{\tau \sqrt{2}} \sqrt{1-\sqrt{1-4 \tau^{2} \omega^{2}}}, \quad \tilde{\Omega}=\frac{1}{\tau \sqrt{2}} \sqrt{1+\sqrt{1-4 \tau^{2} \omega^{2}}}, \quad \tilde{K}=\tau^{2} \tilde{\Omega}^{2}+\tau^{2} \frac{\mathrm{d}^{2}}{\mathrm{~d} t^{2}} .
$$

The resummed projected equation, which makes sense for $\omega<1 /(2 \tau)$, can be quickly obtained by inverting the operator $\tilde{K}$ according to the classical fakeon prescription. The result is

$$
m\left(\frac{\mathrm{d}^{2}}{\mathrm{~d} t^{2}}+\Omega^{2}\right) x=\left\langle F_{\text {ext }}\right\rangle_{\tilde{K}}=\int_{-\infty}^{\infty} \mathrm{d} u \frac{\sin (\tilde{\Omega}|u|)}{2 \tau^{2} \tilde{\Omega}} F_{\text {ext }}(t-u) .
$$

If we expand the average back in powers of $\omega^{2}$, we find

$$
\left\langle F_{\text {ext }}\right\rangle_{\tilde{K}}=\left\langle F_{\text {ext }}\right\rangle_{K}+\tau^{2} \omega^{2}\left(1+\tau^{2} \omega^{2}\right)\left\langle\left\langle F_{\text {ext }}\right\rangle_{K}\right\rangle_{K}+\tau^{4} \omega^{4}\left\langle\left\langle\left\langle F_{\text {ext }}\right\rangle_{K}\right\rangle_{K}\right\rangle_{K}+\mathcal{O}\left(\omega^{6}\right),
$$

which shows that the identity (2.8) is crucial to deal with the multiple averages that lead to the projected equation (2.11) from the unprojected equation (2.10).

The fakeons that are projected away are the solutions of $\tilde{K} x=0$, i.e.

$$
x(t)=C \cos (\tilde{\Omega} t+\varphi) .
$$

The result of the resummation highlights some nontrivial, nonperturbative effects that come into play beyond the convergence radius of the expansion. Indeed, for $\omega>1 /(2 \tau)$ the frequencies $\Omega$ and $\tilde{\Omega}$ become complex and the fakeon projection jumps into another "phase", where all four independent solutions are unacceptable and must be projected away.

In more complicated cases it may be hard to tell what the fakeon projection becomes nonperturbatively. In principle, settling this issue requires knowledge of the nonperturbative sector of quantum field theory. However, workarounds are available in lucky situations, as we show in section 6 .

\section{$3 \quad$ Fakeon projection by asymptotic expansion}

When the potential $V$ contains anharmonic terms, the equations must be treated self consistently. One way to handle the fakeon projection, which we investigate in this section, is by means of an iterative procedure. The projected equations that we obtain are nonpolynomial and must in general be interpreted as asymptotic expansions.

To begin with, let us consider the Lagrangian (2.1) with the potential

$$
V=\frac{m}{2} \omega^{2} x^{2}+\frac{\lambda}{4 !} x^{4} .
$$

The unprojected equation of motion is

$$
m\left(\frac{\mathrm{d}^{2}}{\mathrm{~d} t^{2}}+\tau^{2} \frac{\mathrm{d}^{4}}{\mathrm{~d} t^{4}}+\omega^{2}\right) x=m \tilde{K}\left(\frac{\mathrm{d}^{2}}{\mathrm{~d} t^{2}}+\Omega^{2}\right) x=-\frac{\lambda x^{3}}{3 !} .
$$

We assume $\omega<1 /(2 \tau)$. If we resum the expansion in powers of $\omega^{2}$ as explained in the previous section, we obtain the projected equation

$$
m\left(\frac{\mathrm{d}^{2}}{\mathrm{~d} t^{2}}+\Omega^{2}\right) x=-\frac{\lambda}{3 !}\left\langle x^{3}\right\rangle_{\tilde{K}},
$$

which must still be understood perturbatively in $\lambda$. 
One way to deal with (3.3) is to search for a solution of the form

$$
x(t)=x_{0}(t)+\sum_{n=1}^{\infty} \tilde{\lambda}^{n} x_{n}(t),
$$

where $\tilde{\lambda}=\lambda / m$ and $x_{0}(t)$ solves the homogeneous equation $\ddot{x}_{0}=-\Omega^{2} x_{0}$. We get

$$
\left(\frac{\mathrm{d}^{2}}{\mathrm{~d} t^{2}}+\Omega^{2}\right) x_{1}=-\frac{1}{3 !}\left\langle x_{0}^{3}\right\rangle_{\tilde{K}}, \quad\left(\frac{\mathrm{d}^{2}}{\mathrm{~d} t^{2}}+\Omega^{2}\right) x_{2}=-\frac{1}{2}\left\langle x_{1} x_{0}^{2}\right\rangle_{\tilde{K}},
$$

etc., which can be solved by means of the fakeon averages and the rules outlined before.

Another way is to write a generic expansion for the right-hand side,

$$
\left(\frac{\mathrm{d}^{2}}{\mathrm{~d} t^{2}}+\Omega^{2}\right) x=x \sum_{n=1}^{\infty} \tilde{\lambda}^{n} \tau^{2 n-2} \sum_{k=0}^{n} c_{n, k} x^{2 n-2 k}(\tau \dot{x})^{2 k},
$$

insert it into the unprojected equation (3.2) and determine the unknown coefficients $c_{n, k}$ by matching the monomials. So doing, we can build the projected equation to arbitrarily high orders in $\tilde{\lambda}$. To the first order, we obtain

$$
\left(\frac{\mathrm{d}^{2}}{\mathrm{~d} t^{2}}+\Omega^{2}\right) x=-\frac{\tilde{\lambda} x\left[\left(\tilde{\Omega}^{2}-7 \Omega^{2}\right) x^{2}-6 \dot{x}^{2}\right]}{6 \tau^{2}\left(\tilde{\Omega}^{2}-\Omega^{2}\right)\left(\tilde{\Omega}^{2}-9 \Omega^{2}\right)}+\mathcal{O}\left(\tilde{\lambda}^{2}\right) .
$$

At higher orders we find very involved expressions. For the sake of simplicity, from this point onwards we take $\omega=0$ (which means $\Omega=0, \tilde{\Omega}=1 / \tau$ ). Every result can be generalized straightforwardly to nonvanishing $\omega$. To the third order we obtain

$$
\begin{aligned}
\ddot{x}= & -\frac{\tilde{\lambda} x}{6}\left(x^{2}-6 \tau^{2} \dot{x}^{2}\right)-\frac{\tilde{\lambda}^{2} \tau^{2} x}{12}\left(x^{4}-48 \tau^{2} x^{2} \dot{x}^{2}+372 \tau^{4} \dot{x}^{4}\right) \\
& -\frac{\tilde{\lambda}^{3} \tau^{4} x}{6}\left(x^{6}-156 \tau^{2} x^{4} \dot{x}^{2}+4572 \tau^{4} x^{2} \dot{x}^{4}-31152 \tau^{6} \dot{x}^{6}\right)+\mathcal{O}\left(\tilde{\lambda}^{4}\right) .
\end{aligned}
$$

The truncation of the projected equation to a finite order $n$ in $\tilde{\lambda}$ is polynomial. The expansion is asymptotic and the coefficients grow very fast, although slower than $(4 n)$ !. In this table we give the orders of magnitude of the coefficients $c_{n, 0}$ and $c_{n, n}$ for various values of $n$, which we have computed up to $n=25$ :

\begin{tabular}{|c|c|c|c|c|c}
\hline$n$ & 5 & 10 & 15 & 20 & 25 \\
\hline$c_{n, 0}$ & $10^{0}$ & $10^{6}$ & $10^{13}$ & $10^{22}$ & $10^{32}$ \\
\hline$c_{n, n}$ & $10^{9}$ & $10^{28}$ & $10^{52}$ & $10^{78}$ & $10^{107}$ \\
\hline
\end{tabular}

Note that the expansion we are dealing with does not coincide with the "low-energy" expansion in powers of $\tau^{2}$, which treats the higher-derivative term $\tau^{2} \mathrm{~d}^{2} / \mathrm{d} t^{2}$ as small. Instead, we are expanding in the dimensionless parameter $\tilde{\lambda}$, so each truncation gives a solution that in principle holds for all times. The price we pay is that we have to handle more involved truncations. Indeed, the coefficient $c_{n, k}$, which is multiplied by $\tilde{\lambda}^{n} \tau^{2 n+2 k-2}$, becomes relevant at the $n$th order of the expansion in powers of $\tilde{\lambda}$, but only 


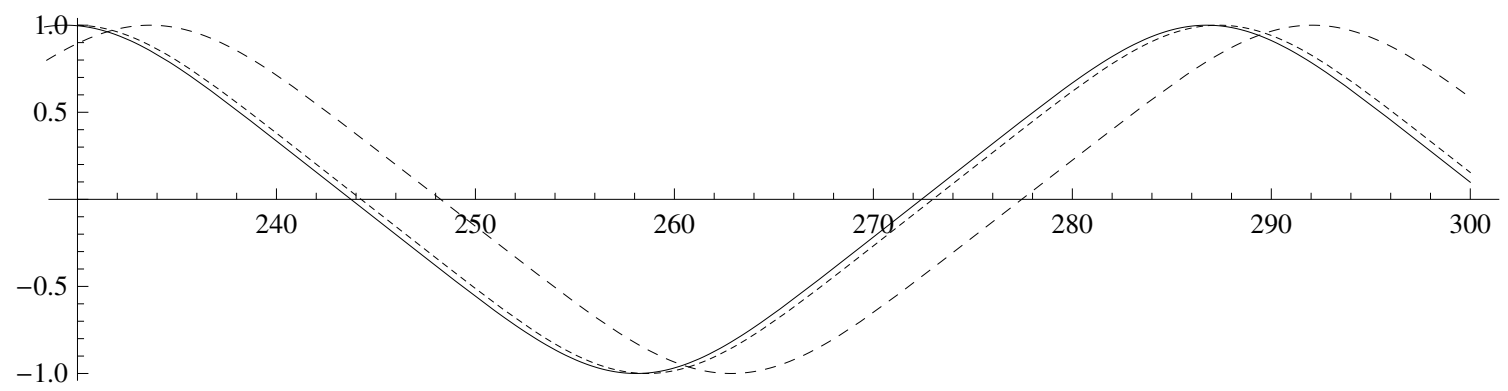

Figure 3. Solution $x(t)$ of the truncated equation (3.5) for $x(0)=1, \dot{x}(0)=0, m=\tau=1$, $\lambda=1 / 10$. The sparsely dashed line is $n=1$. The densely dashed line is $n=2$, while the continuous line is $n=3$. The solution remains stable from $n=3$ to $n=10$.

at the $(n+k+1)$ th order of the expansion in powers of $\tau^{2}$. As we see already from (3.5), the latter grows much more slowly than the former.

The expansion of the projected Lagrangian $\mathcal{L}$ can be worked out in a similar way. We write a generic expansion in $x, \dot{x}$ and determine its coefficients by demanding that the Lagrange equations be equivalent to (3.5). The result is

$$
\frac{\mathcal{L}}{m}=\frac{\dot{x}^{2}}{2}-\frac{\tilde{\lambda} x^{2}}{4 !}\left(x^{2}+12 \tau^{2} \dot{x}^{2}\right)+\frac{\tau^{2} \tilde{\lambda}^{2} x^{2}}{72}\left(x^{4}-54 \tau^{2} x^{2} \dot{x}^{2}+372 \tau^{4} \dot{x}^{4}\right)+\mathcal{O}\left(\tilde{\lambda}^{3}\right) .
$$

The energy $E$ can be obtained from $\mathcal{L}$ or, again, by writing the most general expansion and working out the coefficients that make $\mathrm{d} E / \mathrm{d} t$ vanish on the solutions of (3.5):

$$
\frac{E}{m}=\frac{\dot{x}^{2}}{2}+\frac{\tilde{\lambda} x^{2}}{4 !}\left(x^{2}-12 \tau^{2} \dot{x}^{2}\right)-\frac{\tau^{2} \tilde{\lambda}^{2} x^{2}}{72}\left(x^{4}+54 \tau^{2} x^{2} \dot{x}^{2}-1116 \tau^{4} \dot{x}^{4}\right)+\mathcal{O}\left(\tilde{\lambda}^{3}\right) .
$$

For each truncation to order $n$, the projected equations can be solved numerically. If we compare the solutions for growing $n$, we observe the typical behaviors of the asymptotic solutions. The lowest values of $n$ give results that are acceptable, but not very accurate. Then we find stable results in a certain window $n_{1} \leqslant n \leqslant n_{2}$, which provides the best approximation of the exact solution. Finally, unreliable behaviors appear for $n>n_{2}$. On general gounds, $n_{2}$ is proportional to $1 / \tilde{\lambda}$. Asymptotic expansions cannot be arbitrarily precise, but in several situations the window $n_{1} \leqslant n \leqslant n_{2}$ is precise enough.

For example, with the initial conditions $x(0)=1, \dot{x}(0)=0$ and $m=\tau=1, \lambda=1 / 10$, we find the trajectories of figure 3 . The solution with $n=1$ is not very accurate, while the one with $n=2$ is considerably better. The trajectory remains stable in the window $3 \leqslant n \leqslant 10$. The robust stability is a benefit of the stability of the potential (3.1). With different values of $\lambda$ we find $n_{2} \sim 1 / \lambda$.

\section{The nonperturbative fakeon projection}

The nonperturbative fakeon projection can only follow from the knowledge of the nonperturbative sector of quantum field theory. Indeed, it is easy to show that, when asymptotic expansions are the best we have, at the nonperturbative level the arbitrariness associated with the essential singularities takes us back to the unprojected equations. 
Let $S_{\mathrm{HD}}(\phi, \lambda, \tau)$ denote a higher-derivative action that depends on the fields $\phi^{i}$, $i=1, \ldots N$, and their first $M$ time derivatives. Let $\lambda$ denote the couplings, such that $S_{\mathrm{HD}}(\phi, 0, \tau)$ is free. Let $\tau$ denote the parameters that multiply the higher-derivative corrections, such that $S_{\mathrm{HD}}(\phi, \lambda, 0)$ is the non-higher-derivative action.

We assume that all the degrees of freedom due to the higher derivatives are quantized as fakeons. We focus on the dependence on the time coordinate $t$ and ignore any dependence on the space coordinates $x, y, z$. It is understood that, when we talk about initial or integration "constants", they may be functions of $x, y$ and $z$. We also assume that the fields $\phi^{i}$ are "bosonic", so the field equations depend on $\phi^{i}, \dot{\phi}^{i}$ and $\ddot{\phi}^{i}$ at $\tau=0$.

We have three versions of the classical equations:

(a) the higher-derivative equations

$$
E_{\mathrm{HD}}^{i}(\phi, \lambda, \tau)=0
$$

which are exact, but unprojected; they are satisfied by the acceptable solutions, but also by the fakeon solutions, which must be discarded;

(b) the projected equations

$$
E_{\mathrm{P}}^{i}(\phi, \lambda, \tau)=0
$$

which are understood perturbatively in $\lambda$;

(c) the exact projected equations

$$
E_{\mathrm{PnP}}^{i}(\phi, \lambda, \tau)=0
$$

which can in principle be determined by studying the nonperturbative sector of the parent quantum field theory.

In the example treated above, $(a)$ are (3.2) and $(b)$ are (3.3). We assume that $(c)$ are not known. However, we assume that they exist.

Now, let

$$
\phi^{i}=f^{i}\left(t, \lambda, \tau, c^{i a}\right)
$$

denote the solutions of (4.1), where $c^{i a}$ are the integration constants $(a=1, \ldots M)$ that parametrize the initial conditions. The solutions of the exact projected equations (4.3) are particular cases of (4.4). They have the form

$$
\phi^{i}=f^{i}\left(t, \lambda, \tau, d^{i a}\left(a^{i}, b^{i}, \lambda, \tau\right)\right),
$$

where the constans $d^{i a}$ are not independent, but functions

$$
d^{i a}\left(a^{i}, b^{i}, \lambda, \tau\right)
$$

of $\lambda, \tau$ and $2 N$ independent integration constants $a^{i}, b^{i}$. The solutions of (4.2) are particular cases of (4.5),

$$
\phi^{i}=f^{i}\left(t, \lambda, \tau, c^{i a}\left(a^{i}, b^{i}, \lambda, \tau\right)\right),
$$


where the functions $c^{i a}\left(a^{i}, b^{i}, \lambda, \tau\right)$ are only known as asymptotic expansions in powers of $\lambda$ and coincide with the asymptotic expansions of (4.6).

The difference $d^{i a}\left(a^{i}, b^{i}, \lambda, \tau\right)-c^{i a}\left(a^{i}, b^{i}, \lambda, \tau\right)$ is made of essential singularities for $\lambda \rightarrow 0$, which cannot be worked out from the sole knowledge of $(a)$ and $(b)$. If we attempt a resummation (with the Borel method, for example, when applicable), the solution will unlikely satisfy (4.1). The space of functions that have the same asymptotic expansions and satisfy (4.1) at the same time obviously coincides with the space of unprojected solutions (4.4).

This means that, unless we have direct knowledge about the nonperturbative sector of the parent quantum field theory, we cannot write "exact" classical field equations and mostly have to work with their perturbative form.

However, workarounds may be available in special cases by means of resummations. Even in quantum field theory we have example of exact results that can be derived form the perturbative expansion. We mention the anomalies (which are one-loop exact), the renormalization group flow (which allows us to resum the leading logs, the next-to-leading logs, etc.), the particle self-energies, obtained by resumming the bubble diagrams (which give the particle lifetimes, among other things), and so on. Similarly, there are cases where, in spite of the difficulties stressed in this section, we can get to the exact projected solutions (4.5) in quantum gravity. In the following sections we describe some important examples.

\section{The classical limit of quantum gravity}

Before proceeding, we briefly recall the basic aspects of the classicization of quantum gravity. At the conceptual level, it is convenient to work with the non-higher-derivative interim classical action (1.2). The field equations of the metric read

$$
R^{\mu \nu}-\frac{1}{2} g^{\mu \nu} R=\frac{\kappa^{2}}{\zeta}\left[\mathrm{e}^{3 \kappa \phi} f T_{\mathfrak{m}}^{\mu \nu}\left(\tilde{g} \mathrm{e}^{\kappa \phi}, \Phi\right)+f T_{\phi}^{\mu \nu}(\tilde{g}, \phi)+T_{\chi}^{\mu \nu}(g, \chi)\right],
$$

where $T_{A}^{\mu \nu}(g)=-(2 / \sqrt{-g})\left(\delta S_{A}(g) / \delta g_{\mu \nu}\right)$ are the energy-momentum tensors $(A=\mathfrak{m}, \phi$, $\chi)$ and $f=\sqrt{\operatorname{det} \tilde{g}_{\rho \sigma} / \operatorname{det} g_{\alpha \beta}}$. The field equations of the fakeons $\phi$ and $\chi_{\mu \nu}$ are [14]

$$
\begin{aligned}
-\frac{1}{\sqrt{-\tilde{g}}} \partial_{\mu}\left(\sqrt{-\tilde{g}} \tilde{g}^{\mu \nu} \partial_{\nu} \phi\right)-\frac{m_{\phi}^{2}}{\kappa}\left(\mathrm{e}^{\kappa \phi}-1\right) \mathrm{e}^{\kappa \phi} & =\frac{\kappa \mathrm{e}^{3 \kappa \phi}}{3 \zeta} T_{\mathfrak{m}}^{\mu \nu}\left(\tilde{g} \mathrm{e}^{\kappa \phi}, \Phi\right) \tilde{g}_{\mu \nu}, \\
\frac{1}{\sqrt{-g}} \frac{\delta S_{\chi}(g, \chi)}{\delta \chi_{\mu \nu}} & =\mathrm{e}^{3 \kappa \phi} f T_{\mathfrak{m}}^{\mu \nu}\left(\tilde{g} \mathrm{e}^{\kappa \phi}, \Phi\right)+f T_{\phi}^{\mu \nu}(\tilde{g}, \phi) .
\end{aligned}
$$

Let $\langle\phi\rangle$ and $\left\langle\chi_{\mu \nu}\right\rangle$ denote the solutions of the equations (5.2), obtained with the half sum of the retarded and advanced Green functions. The projected field equations are (5.1), once $\phi$ and $\chi_{\mu \nu}$ are replaced by $\langle\phi\rangle$ and $\left\langle\chi_{\mu \nu}\right\rangle$. They can also be derived as Lagrange equations of the finalized classical action

$$
\mathcal{S}_{\mathrm{QG}}^{\mathrm{GFF}}(g, \Phi)=S_{\mathrm{H}}(g)+S_{\chi}(g,\langle\chi\rangle)+S_{\phi}(\bar{g},\langle\phi\rangle)+S_{\mathfrak{m}}\left(\bar{g} \mathrm{e}^{\kappa\langle\phi\rangle}, \Phi\right),
$$

where $\bar{g}_{\mu \nu}=g_{\mu \nu}+2\left\langle\chi_{\mu \nu}\right\rangle$. 
As said, we have to understand the projection perturbatively and deal with the issues explained in the previous sections. In the next sections we study the resummation of the perturbative projection in the case of the FLRW solution. At the practical level, it is more convenient to work with the interim action (1.1), but in section 7 we give details on how to obtain the same results by working with (1.2).

\section{The classicization of the FLRW solution}

It is often convenient to search for solutions of the field equations starting from an ansatz, as in the case of the FLRW metric. However, in general, it is not legitimate to insert the ansatz directly into the action and work out the Lagrange equations of the so-obtained reduced action. Indeed, the ansatz reduces the space of configurations. A minimum, or more generally extremum, of the action on the reduced space of configurations is not guaranteed to be a minimum or extremum on the full space.

However, under certain conditions it is possible to obtain the correct equations of motion by applying the variational principle to the reduced action. We derive the key properties to achieve this goal and then apply the method of the reduced action to the FLRW ansatz.

\subsection{Method of the reduced action}

Consider an action $S(\phi)$ depending on the fields $\phi^{i}, i=1, \ldots N$. The Lagrange equations are

$$
\frac{\delta S}{\delta \phi^{i}}=0
$$

Consider an ansatz

$$
\phi^{i}=f^{i}(\varphi)
$$

that expresses the fields $\phi^{i}$ in terms of a reduced set of fields $\varphi^{\alpha}, \alpha=1, \ldots M$, with $M<N$. The reduced action is then

$$
S_{r}(\varphi)=S(f(\varphi))
$$

and its field equations read

$$
0=\frac{\delta S_{r}(\varphi)}{\delta \varphi^{\alpha}}=\left.\frac{\delta S}{\delta \phi^{i}}\right|_{\phi=f(\varphi)} \frac{\delta f^{i}(\varphi)}{\delta \varphi^{\alpha}}
$$

Now, assume that

(i) the $M$ equations (6.3) are independent, and

(ii) $M$ equations (6.1) are independent and the other $N-M$ equations (6.1) are algebraic relations among the $M$ independent ones.

Then, the equations (6.1) are equivalent to the equations (6.3) derived from the reduced action $S_{r}$.

Typically, point (ii) can be established by means of symmetry arguments and other properties of the ansatz. Point $(i)$ is easy to check directly. 
If the relations mentioned in point (ii) happen to be differential instead of algebraic, further assumptions must be advocated to obtain the right set of equations after the reduction.

\subsection{The FLRW metric}

Now we apply the method of the reduced action to the FLRW metric, which we parametrize as

$$
\mathrm{d} s^{2}=g_{\mu \nu} \mathrm{d} x^{\mu} \mathrm{d} x^{\nu}=b^{2}(t) \mathrm{d} t^{2}-a^{2}(t) \mathrm{d} \sigma^{2},
$$

where, in spherical polar coordinates,

$$
\mathrm{d} \sigma^{2}=\frac{\mathrm{d} r^{2}}{1-k r^{2}}+r^{2} \mathrm{~d} \theta^{2}+r^{2} \sin ^{2} \theta \mathrm{d} \phi^{2} .
$$

The lapse function $b(t)$ is inserted to meet the requirements explained above and keep track of the time reparametrizations. Indeed, we know that the FLRW ansatz reduces the field equations to two independent ones, so we need two independent functions $a(t)$ and $b(t)$ to have a meaningful reduced action $S_{r}$. We can set $b(t) \equiv 1$ after applying the variational principle to $S_{r}$.

Under the usual assumptions of homogeneity and isotropy, the matter stress tensor is

$$
\left(T_{\mathfrak{m}}\right)_{\mu}^{\nu}=\rho(t) \delta_{0}^{\nu} \delta_{\mu}^{0}-p(t) \delta_{i}^{\nu} \delta_{\mu}^{i}
$$

where $\rho$ is the energy density and $p$ is the pressure, $i=1,2,3$ being a space index. Then the reduced version of the action (1.1) of quantum gravity coupled to matter reads

$$
S_{\mathrm{QG}} \rightarrow-\frac{1}{16 \pi G} \frac{r^{2} \sin \theta}{\sqrt{1-k r^{2}}} \int \mathrm{d} t a^{3} b \mathcal{R}\left(1-\frac{\mathcal{R}}{6 m_{\phi}^{2}}\right)+S_{\mathfrak{m}}
$$

where $m_{\phi}^{2}=\zeta / \xi$ and the Ricci curvature for the ansatz (6.4) is

$$
\mathcal{R}=-6\left(\frac{\ddot{a}}{a b^{2}}+\frac{\dot{a}^{2}}{a^{2} b^{2}}-\frac{\dot{a} \dot{b}}{a b^{3}}+\frac{k}{a^{2}}\right) .
$$

The arrow in formula (6.6) and in the formulas below means that we ignore the integrals on $r$ and the angles $\theta$ and $\varphi$, which give an overall (infinite) factor that can be dropped for the purpose of applying the variational principle.

Note that the $\alpha$-dependent terms of (1.1) cancel out, because they are proportional to the square of the Weyl tensor $C^{\mu \nu \rho \sigma}$, up to a total derivative, and $C^{\mu \nu \rho \sigma}$ vanishes identically for the metric (6.4).

We do not have a well-defined expression for $S_{\mathfrak{m}}$, with the stress tensor (6.5). However, the infinitesimal variation $\delta S_{\mathfrak{m}}$ is enough for our purposes. It reads

$$
\delta S_{\mathfrak{m}}=-\frac{1}{2} \int \mathrm{d}^{4} x \sqrt{-g}\left(T_{\mathfrak{m}}\right)_{\mu}^{\nu} g^{\mu \rho} \delta g_{\nu \rho} \rightarrow \frac{r^{2} \sin \theta}{\sqrt{1-k r^{2}}} \int \mathrm{d} t a^{2}(3 p b \delta a-\rho a \delta b)
$$


If we vary the reduced action with respect to $a$ and $b$ and then set $b(t) \equiv 1$, we obtain the unprojected equations

$$
\Sigma\left(\frac{\ddot{a}}{a}+\frac{\dot{a}^{2}}{a^{2}}+\frac{k}{a^{2}}\right)=\frac{4 \pi G}{3}(\rho-3 p), \quad \Upsilon\left(\frac{\ddot{a}}{a}-\frac{\dot{a}^{2}}{a^{2}}-\frac{k}{a^{2}}\right)=-4 \pi G(\rho+p),
$$

where $\Sigma$ and $\Upsilon$ are the operators

$$
\Sigma=1+\frac{1}{m_{\phi}^{2}}\left(3 \frac{\dot{a}}{a}+\frac{\mathrm{d}}{\mathrm{d} t}\right) \frac{\mathrm{d}}{\mathrm{d} t}, \quad \Upsilon=\Sigma+\frac{2}{m_{\phi}^{2}}\left[\frac{k}{a^{2}}+3 \frac{\mathrm{d}}{\mathrm{d} t}\left(\frac{\dot{a}}{a}\right)\right] .
$$

The continuity equation

$$
\dot{\rho}+3(\rho+p) \frac{\dot{a}}{a}=0
$$

is the same as usual. It follows from the conservation of the stress-energy tensor and can be checked by solving (6.8) for $\rho$ and $p$.

It is easy to verify that the equations (6.8) match those obtained by inserting the ansatz (6.4) with $b(t) \equiv 1$ into the field equations of (1.1) (which can be found for example in ref. [14]), as guaranteed by the method of the reduced action.

\subsection{Projection}

Since the left-hand sides of the equations (6.8) factorize the operators $\Sigma$ and $\Upsilon$, the resummed fakeon projection is straightforward. If we multiply (6.8) by $\Sigma^{-1}$ and $\Upsilon^{-1}$, defined by means of the classical fakeon prescription, we obtain the projected equations

$$
\begin{aligned}
& \frac{\ddot{a}}{a}+\frac{\dot{a}^{2}}{a^{2}}+\frac{k}{a^{2}}=\frac{4 \pi G}{3}\langle\rho-3 p\rangle_{\Sigma}, \\
& \frac{\ddot{a}}{a}-\frac{\dot{a}^{2}}{a^{2}}-\frac{k}{a^{2}}=-4 \pi G\langle\rho+p\rangle_{\Upsilon},
\end{aligned}
$$

where the fakeon averages are defined in (2.4).

For some purposes, it is convenient to define a modified energy density $\tilde{\rho}$ and a modified pressure $\tilde{p}$ as

$$
\begin{aligned}
& \tilde{\rho}=\frac{1}{4}\langle\rho-3 p\rangle_{\Sigma}+\frac{3}{4}\langle\rho+p\rangle_{\Upsilon}, \\
& \tilde{p}=\frac{1}{4}\langle\rho+p\rangle_{\Upsilon}-\frac{1}{4}\langle\rho-3 p\rangle_{\Sigma},
\end{aligned}
$$

and rearrange (6.11) and (6.12) in forms that match the usual Friedmann equations:

$$
\begin{aligned}
\frac{\dot{a}^{2}}{a^{2}}+\frac{k}{a^{2}} & =\frac{8 \pi G}{3} \tilde{\rho}, \\
2 \frac{\ddot{a}}{a}+\frac{\dot{a}^{2}}{a^{2}}+\frac{k}{a^{2}} & =-8 \pi G \tilde{p} .
\end{aligned}
$$

Adding the derivative of (6.15) to a suitable linear combination of the two equations, it is easy to get the second continuity equation

$$
\frac{\mathrm{d} \tilde{\rho}}{\mathrm{d} t}+3 \frac{\dot{a}}{a}(\tilde{\rho}+\tilde{p})=0,
$$

satisfied by the modified energy density and pressure. 
Depending on the problem at hand, the fakeon projection encoded in the equations (6.11) and (6.12) may or may not be the final, exact one. It is exact in some important cases, which include the vacuum energy density, radiation and their combination. It is not exact in other cases, which include dust (cold matter). There, however, approximate solutions are enough for most purposes.

Vacuum energy density. Now we show that in the case of the vacuum energy the solutions of the projected equations coincide with the solutions of the Friedmann equations that follow from Einstein gravity.

The equation of state is $p=-\rho$, so the continuity equation (6.10) gives $\rho=\rho_{0}=$ constant. It is convenient to start by solving (6.12), since its right-hand side vanishes. The solution reads

$$
a(t)=\mathrm{e}^{\sigma t}+\frac{k}{4 \sigma^{2}} \mathrm{e}^{-\sigma t},
$$

where $\sigma$ is another constant. The third integration constant has been absorbed into a time translation.

To study (6.11), note that (6.18) and $\rho=\rho_{0}$ imply $\Sigma \rho=\rho$. Thus, we also have $\langle\rho\rangle_{\Sigma}=\rho$ and, from (6.13)-(6.14), $\tilde{\rho}=\rho, \tilde{p}=p=-\rho$. Then, equation (6.11) gives a relation between the two constants $\rho_{0}$ and $\sigma$, which reads

$$
\rho_{0}=\frac{3 \sigma^{2}}{8 \pi G} \text {. }
$$

Radiation. Similar conclusions hold in the case of radiation, where $p=\rho / 3$. The continuity equation (6.10) gives

$$
\rho(t)=\frac{\rho_{0}^{\prime}}{a^{4}},
$$

where $\rho_{0}^{\prime}$ is constant. Solving (6.11), whose right-hand side vanishes, we get

$$
a(t)=\sqrt{t\left(\sigma^{\prime}-k t\right)},
$$

up to a time translation, $\sigma^{\prime}$ being another constant.

Using (6.20) and (6.21) we easily find $\Upsilon \rho=\rho$, so $\langle\rho\rangle_{\Upsilon}=\rho, \tilde{\rho}=\rho, \tilde{p}=p=\rho / 3$. Then, equation (6.12) gives

$$
\rho_{0}^{\prime}=\frac{3 \sigma^{\prime 2}}{32 \pi G}
$$

Combination of radiation and vacuum energy density. Consider the equation of state

$$
p=\frac{\rho}{3}+p_{0}=\frac{1}{3}\left(\rho-4 \rho_{0}\right),
$$

where $\rho_{0}$ and $p_{0}=-4 \rho_{0} / 3$ are constants. The interesting feature of $(6.22)$ is that it allows us to treat the combination of radiation and the vacuum energy density, which can be useful to study inflation. As before, we can solve the projected equations exactly, since (6.13) and (6.14) give $\tilde{p}=\left(\tilde{\rho}-4 \rho_{0}\right) / 3$. For convenience, we write $\rho_{0}=3 \sigma^{2} /(8 \pi G)$. The continuity equation (6.17) gives

$$
\tilde{\rho}(t)=\frac{3}{8 \pi G}\left(\sigma^{2}+\frac{\sigma^{\prime 2}}{4 a^{4}}\right)
$$


where $\sigma^{\prime}$ is constant. Inserting this solution into (6.15), we get

$$
a(t)=\sqrt{\frac{\sinh (\sigma t)}{\sigma}\left(\sigma^{\prime} \cosh (\sigma t)-\frac{k}{\sigma} \sinh (\sigma t)\right)},
$$

up to a time translation. We can check that (6.24) satisfies (6.16) identically. When $\sigma \rightarrow 0$ we retrieve (6.21). For $\sigma^{\prime} \rightarrow 0$ and $k<0$, we obtain a time-translated version of (6.18).

We can find the energy density $\rho$ from the second unprojected equation of formula (6.8). The result is very similar to $(6.23)$,

$$
\rho(t)=\frac{3}{8 \pi G}\left(\sigma^{2}+\frac{\sigma^{\prime \prime 2}}{4 a^{4}}\right), \quad \sigma^{\prime \prime 2}=\sigma^{\prime 2}\left(1+\frac{4 \sigma^{2}}{m_{\phi}^{2}}\right),
$$

the only change being the coefficient of the contribution due to the radiation. This is also the only correction to the result obtained from the Einstein equations.

General case. In general, if we assume the equation of state $p=w \rho$, the modified pressure and density satisfy an $a$-dependent differential equation of state, which reads

$$
\tilde{p}=w \tilde{\rho}-\frac{(1+w)(1-3 w)}{3(1+w) \Sigma+(1-3 w) \Upsilon} \Delta \tilde{\rho}
$$

where $\Delta=\Upsilon-\Sigma$ and the reciprocal operator that appears here has to be defined by means of the fakeon prescription (2.4).

The continuity equation (6.10) gives the usual relation

$$
\rho(t)=\frac{3 \sigma^{\prime 2}}{32 \pi G} \frac{1}{a^{3(1+w)}},
$$

where $\sigma^{\prime}$ is constant. It is convenient to introduce a function $u(t)$ by writing

$$
a(t)=\left[3 \sigma^{\prime}(1+w) u(t) / 4\right]^{2 /(3(1+w))} .
$$

Then the unprojected equations (6.8) give, in the simple case $k=0$,

$$
m_{\phi}^{2}\left(1-\dot{u}^{2}\right)=2 \dot{u} \dddot{u}-\ddot{u}^{2}-\frac{4 w}{1+w} \frac{\dot{u}^{2} \ddot{u}}{u}-\frac{1-3 w}{1+w} \frac{\dot{u}^{4}}{u^{2}} .
$$

The fakeon projection of this equation is rather hard, since it contains no parameter that we can use to approach the problem perturbatively, other than $\tau \equiv 1 / m_{\phi}$. If we expand in powers of $\tau$ we obtain the usual low-energy expansion,

$$
u(t)=t\left[1-\frac{1-3 w}{2(1+w)} \frac{1}{m_{\phi}^{2} t^{2}}+\frac{13+34 w-219 w^{2}}{24(1+w)^{2}} \frac{1}{m_{\phi}^{4} t^{4}}+\mathcal{O}\left(\frac{1}{m_{\phi}^{6} t^{6}}\right)\right] .
$$

As in section 3 , the series is asymptotic and the numerical coefficients grow very fast. For example, in the case of dust $(w=0)$ the coefficient of $1 /\left(m_{\phi} t\right)^{14}$ is of order $10^{8}$. Depending on the values of $m_{\phi} t$, various terms of the asymptotic expansion may offer a stable, satisfactory approximation of the exact solution. 
The reason why the projections that appear in (6.11) and (6.12), which are resummed versions of those obtained by expanding around flat space, are not the exact projections for the problem we are dealing with, is that the solutions for $a(t), \rho(t)$ and $p(t)$ must be worked out self-consistently. The equations would be exact if $a(t)$ had to be found for given $\rho(t)$ and $p(t)$ (see comments below).

We expect that the masses of the fakeons $\chi_{\mu \nu}$ and (possibly) $\phi$ have values that are much smaller than the Planck mass. On general grounds, they could be around $10^{12} \mathrm{GeV}$ [4]. If that is the case, the value of the parameter $\tau \sim 1 / m_{\chi} \sim 1 / m_{\phi}$, which multiplies the higher time derivative $\mathrm{d} / \mathrm{d} t$, is around $10^{-36} \mathrm{~s}$. We know that the first moments of the life of the universe were dominated by radiation, with a crossover to matter dominance at $t \sim 5 \cdot 10^{4}$ years. The matter dominated epoch lasted about $10^{10}$ years, followed by the dark energy era. Thus, an exact treatment of the matter dominated epoch is not strictly necessary in cosmology and the first few orders of (6.27) can be enough for most purposes. As shown previously, the radiation dominated era can be treated exactly, even in superposition with the vacuum energy density.

Other cases where the projection can be worked out exactly. We conclude by pointing out other situations where the projection can be handled exactly. The first case is when we need to find the FLRW metric for given sources, i.e. $\rho$ and $p$ do not have to be determined self-consistently, but are given functions, known from the start. Then, the projection encoded in equations (6.11) and (6.12) is exact. The solutions do not coincide with those predicted by Einstein gravity and averages similar to those found in section 2 appear. The case is to some extent similar to the case of the harmonic oscillator with an external force, whose fakeon projection is encoded in formula (2.11).

We stress that, on the contrary, when $\rho, p$ have to be solved self-consistently together with the metric, the projection contained in the equations (6.11) and (6.12) must still be understood perturbatively. The iterative methods of section 3 can be used to work out the asymptotic expansions of the solutions, which may be satisfactory for some purposes. An example is the FLRW metric for nonrelativistic matter.

The second case where we can handle the fakeon projection exactly is when for some reason we are given an equation of state expressing $\tilde{p}$ as a function of $\tilde{\rho}$ only. Then, the problem of solving the equations (6.15) and (6.16), with the help of (6.17), matches the problem of solving the Friedmann equations of Einstein gravity.

One may wonder whether it is possible to make the fakeon averages effectively disappear by redefining the density and pressure everywhere, so that $\tilde{\rho}$ and $\tilde{p}$ describe the quantities we really observe or measure, instead of $\rho$ and $p$. In general, it is not legitimate to do so, but in some cases, depending on the data available to us, we may have no other option. More precisely, the relations (6.13) and (6.14) between $\tilde{\rho}, \tilde{p}$ and $\rho, p$ depend on the particular problem we deal with, to the extent that they contain the metric and the ansatz we are using. Other problems may lead to different formulas for the modified quantities $\tilde{\rho}$ and $\tilde{p}$. Moreover, different interactions, such as the electromagnetic ones, are sensitive to the unmodified $\rho$ and $p$. Thus, it possible to probe the relations between $\tilde{\rho}, \tilde{p}$ and $\rho, p$ by comparing different physical situations. However, when these comparisons are 
out of reach, maybe because not enough data are available, it may be impossible to tell that equations (6.15) and (6.16) are actually descendants of the parent equations (6.11) and (6.12).

A similar conclusion extends to the problem of detecting the violations of microcausality. Unless we are able to cross check different physical situations, it may be impossible to uncover the violation, because it may be easily hidden inside redefinitions of the quantities we measure.

\section{Non-higher derivative approach to the FLRW solution}

For completeness, we report how the solutions are worked out from the action (1.2). We start from the ansatz

$$
\begin{aligned}
& g_{\mu \nu} \mathrm{d} x^{\mu} \mathrm{d} x^{\nu}=\bar{b}^{2}(t) \mathrm{d} t^{2}-\bar{a}^{2}(t) \mathrm{d} \sigma^{2}, \\
& \chi_{\mu \nu} \mathrm{d} x^{\mu} \mathrm{d} x^{\nu}=d(t) \mathrm{d} t^{2}-e(t) \mathrm{d} \sigma^{2}, \quad \phi=\phi(t) .
\end{aligned}
$$

With this choice, we have the right amount of independent functions to derive the field equations by means of the reduced action approach. Alternatively, we can insert the ansatz directly into (5.1) and (5.2).

Anticipating the result, it is convenient to define

$$
\bar{b}^{2}=B^{2}-2 d, \quad \bar{a}^{2}=A^{2}-2 e, \quad A=a \mathrm{e}^{-\kappa \phi / 2}, \quad B=b \mathrm{e}^{-\kappa \phi / 2} .
$$

The metric that effectively couples to matter reads

$$
\tilde{g}_{\mu \nu} \mathrm{e}^{\kappa \phi} \mathrm{d} x^{\mu} \mathrm{d} x^{\nu}=b^{2}(t) \mathrm{d} t^{2}-a^{2}(t) \mathrm{d} \sigma^{2},
$$

where $\tilde{g}_{\mu \nu}=g_{\mu \nu}+2 \chi_{\mu \nu}$.

Now we study the $\bar{a}, \bar{b}, \phi, d$ and $e$ field equations, starting from the $\phi$ one, which reads

$$
\Sigma\left(1-\mathrm{e}^{-\kappa \phi}\right)=-\frac{8 \pi G}{3 m_{\phi}^{2}}(\rho-3 p) .
$$

It can be projected straightforwardly, leading to

$$
1-\mathrm{e}^{-\kappa \phi}=-\frac{8 \pi G}{3 m_{\phi}^{2}}\langle\rho-3 p\rangle_{\Sigma}
$$

If we set

$$
\mathrm{e}^{-\kappa \phi}=1-\frac{\mathcal{R}}{3 m_{\phi}^{2}}
$$

where $\mathcal{R}$ is still given by (6.7), equation (7.3) becomes equivalent to equation (6.11).

Since the FLRW metric has a vanishing Weyl tensor, the functions $d(t)$ and $e(t)$ should make the $\alpha$ dependence disappear from the field equations. This goal is achieved by choosing

$$
d(t)=\frac{1}{m_{\chi}^{2}}\left(\frac{\dot{A}^{2}}{A^{2}}-2 \frac{\ddot{A}}{A}+2 \frac{\dot{A} \dot{B}}{A B}+k \frac{B^{2}}{A^{2}}\right), \quad e(t)=-\frac{1}{m_{\chi}^{2}}\left(\frac{\dot{A}^{2}}{B^{2}}+k\right),
$$


where $m_{\chi}^{2}=\zeta / \alpha$. Once we set $b(t) \equiv 1$, we can drop the $\chi_{\mu \nu}$ field equations, obtained from the variations with respect to $d$ and $e$, since it is easy to prove that they are equivalent to the equations obtained from $\bar{a}$ and $\bar{b}$. At the end, the equations (5.1) coincide with (6.8) and can be projected as before, leading to (6.11) and (6.12).

In the case of radiation, (7.4) gives $\phi=0$, while in the case of the vacuum energy density we obtain

$$
\phi=-\frac{1}{\kappa} \ln \left(1+\frac{4 \sigma^{2}}{m_{\phi}^{2}}\right),
$$

where $\sigma$ is the constant appearing in (6.18). Formula (7.5) also holds in the case of radiation combined with the vacuum energy density. Conversely, if we start from the ansatz $\phi=$ constant, equation (7.2) implies $\rho-3 p=$ constant, which is the equation of state of the combination of radiation and the vacuum energy density.

We see that by extending the standard FLRW ansatz (6.4) to (7.1), the presence of the $\chi_{\mu \nu}$ does not affect the solution. It is conceivable that many results obtained in inflationary cosmology [42] can be extended to the full theory of quantum gravity studied here, which has the advantage of being renormalizable.

\section{Conclusions}

When fakeons are present, the starting, local classical action is just an interim one. The true classical action emerges only at the very end, after the quantization, by means of a process of classicization of the quantum theory. The reason is that the fakeon prescription is not classical, but emerges from the loop corrections.

Quantum field theory is formulated perturbatively, so the classicization is also perturbative. The consequences of this fact are quite striking: instead of having complete, exact classical equations, we deal with the typical problems of quantum field theory, even if we work at the classical level. These include the appearance of asymptotic series (when we write the equations, not just when we search for their solutions) and possibly important roles played by the nonperturbative corrections. As far as we know, this backlash of the quantization on the classical limit is unprecedented.

We have investigated the problems related to the resummation of the perturbative expansion associated with the fakeon projection and applied the results to the FLRW metric in quantum gravity. In some cases (like the vacuum energy, radiation and the combination of the two), the fakeon projection can be resummed to all orders. In more general cases, which include dust, asymptotic series are generated and nonperturbative effects may come into play. The implications on the very early stages of the big bang remain to be explored.

\section{Acknowledgments}

We are grateful to U. Aglietti, L. Bracci, M. Piva and T. Morris for useful discussions. 
Open Access. This article is distributed under the terms of the Creative Commons Attribution License (CC-BY 4.0), which permits any use, distribution and reproduction in any medium, provided the original author(s) and source are credited.

\section{References}

[1] D. Anselmi, On the quantum field theory of the gravitational interactions, JHEP 06 (2017) 086 [arXiv: 1704.07728] [INSPIRE].

[2] D. Anselmi, Fakeons And Lee-Wick Models, JHEP 02 (2018) 141 [arXiv:1801.00915] [INSPIRE].

[3] D. Anselmi and M. Piva, The Ultraviolet Behavior of Quantum Gravity, JHEP 05 (2018) 027 [arXiv: 1803.07777] [INSPIRE].

[4] D. Anselmi and M. Piva, Quantum Gravity, Fakeons And Microcausality, JHEP 11 (2018) 021 [arXiv: 1806.03605 ] [INSPIRE].

[5] D. Anselmi and M. Piva, A new formulation of Lee-Wick quantum field theory, JHEP 06 (2017) 066 [arXiv : 1703.04584] [INSPIRE].

[6] R.E. Cutkosky, Singularities and discontinuities of Feynman amplitudes, J. Math. Phys. 1 (1960) 429 [INSPIRE].

[7] M.J.G. Veltman, Unitarity and causality in a renormalizable field theory with unstable particles, Physica 29 (1963) 186 [INSPIRE].

[8] M.E. Peskin and D.V. Schroeder, An introduction to quantum field theory, Addison-Wesley, Reading, U.S.A, (1995), Chapter 7, section 3.

[9] T.D. Lee and G.C. Wick, Negative Metric and the Unitarity of the S Matrix, Nucl. Phys. B 9 (1969) 209 [INSPIRE].

[10] T.D. Lee and G.C. Wick, Finite Theory of Quantum Electrodynamics, Phys. Rev. D 2 (1970) 1033 [INSPIRE].

[11] N. Nakanishi, Lorentz noninvariance of the complex-ghost relativistic field theory, Phys. Rev. D 3 (1971) 811 [INSPIRE].

[12] R.E. Cutkosky, P.V. Landshoff, D.I. Olive and J.C. Polkinghorne, A non-analytic S matrix, Nucl. Phys. B 12 (1969) 281 [inSPIRE].

[13] B. Grinstein, D. O'Connell and M.B. Wise, Causality as an emergent macroscopic phenomenon: The Lee-Wick O(N) model, Phys. Rev. D 79 (2009) 105019 [arXiv:0805.2156] [INSPIRE].

[14] D. Anselmi, Fakeons, Microcausality And The Classical Limit Of Quantum Gravity, Class. Quant. Grav. 36 (2019) 065010 [arXiv: 1809.05037] [INSPIRE].

[15] K.S. Stelle, Renormalization of Higher Derivative Quantum Gravity, Phys. Rev. D 16 (1977) 953 [INSPIRE].

[16] I.G. Avramidi and A.O. Barvinsky, Asymptotic freedom in higher derivative quantum gravity, Phys. Lett. 159B (1985) 269 [INSPIRE].

[17] N. Ohta, R. Percacci and A.D. Pereira, Gauges and functional measures in quantum gravity II: Higher derivative gravity, Eur. Phys. J. C 77 (2017) 611 [arXiv:1610.07991] [INSPIRE].

[18] A. Salvio and A. Strumia, Agravity, JHEP 06 (2014) 080 [arXiv:1403.4226] [INSPIRE]. 
[19] A. Salvio and A. Strumia, Agravity up to infinite energy, Eur. Phys. J. C 78 (2018) 124 [arXiv: 1705.03896] [INSPIRE].

[20] D. Anselmi, The correspondence principle in quantum field theory and quantum gravity, PhilSci 15287, OSF preprints https://doi.org/10.31219/osf.io/d2nj7 Preprints 2018110213 hal-01900207.

[21] M.B. Green, J.H. Schwarz and E. Witten, Superstring Theory I 83 II, Cambridge University Press, (1987).

[22] J. Polchinski, String Theory I \& II, Cambridge University Press, (1998).

[23] K. Becker, M. Becker and J. Schwarz, String theory and M-theory: A modern introduction, Cambridge University Press, (2007).

[24] R. Blumenhagen, D. Lust and S. Theisen, Basic Concepts of String Theory, Springer Verlag, (2012).

[25] A. Ashtekar ed.: 100 years of relativity. Space-time structure: Einstein and beyond, World Scientific, (2005).

[26] C. Rovelli, Quantum Gravity, Cambridge University Press, (2004).

[27] T. Thiemann, Modern canonical quantum general relativity, Cambridge University Press, (2007).

[28] J.M. Maldacena, The large $N$ limit of superconformal field theories and supergravity, Int. J. Theor. Phys. 38 (1999) 1113 [hep-th/9711200] [INSPIRE].

[29] S.S. Gubser, I.R. Klebanov and A.M. Polyakov, Gauge theory correlators from noncritical string theory, Phys. Lett. B 428 (1998) 105 [hep-th/9802109] [INSPIRE].

[30] E. Witten, Anti-de Sitter space and holography, Adv. Theor. Math. Phys. 2 (1998) 253 [hep-th/9802150] [INSPIRE].

[31] V.E. Hubeny, The AdS/CFT Correspondence, Class. Quant. Grav. 32 (2015) 124010 [arXiv: 1501.00007] [INSPIRE].

[32] H.W. Hamber, Quantum Gravity on the Lattice, Gen. Rel. Grav. 41 (2009) 817 [arXiv: 0901.0964] [INSPIRE].

[33] S. Weinberg, Ultraviolet divergences in quantum theories of gravitation, in An Einstein centenary survey, S. Hawking and W. Israel eds., Cambridge University Press, Cambridge (1979), p. 790.

[34] O. Lauscher and M. Reuter, Ultraviolet fixed point and generalized flow equation of quantum gravity, Phys. Rev. D 65 (2002) 025013 [hep-th/0108040] [INSPIRE].

[35] O. Lauscher and M. Reuter, Flow equation of quantum Einstein gravity in a higher derivative truncation, Phys. Rev. D 66 (2002) 025026 [hep-th/0205062] [INSPIRE].

[36] K. Falls, C.R. King, D.F. Litim, K. Nikolakopoulos and C. Rahmede, Asymptotic safety of quantum gravity beyond Ricci scalars, Phys. Rev. D 97 (2018) 086006 [arXiv:1801.00162] [INSPIRE].

[37] M.R. Douglas, The statistics of string/M theory vacua, JHEP 05 (2003) 046 [hep-th/0303194] [INSPIRE].

[38] S. Ashok and M.R. Douglas, Counting flux vacua, JHEP 01 (2004) 060 [hep-th/0307049] [INSPIRE]. 
[39] D. Anselmi, Properties Of The Classical Action Of Quantum Gravity, JHEP 05 (2013) 028 [arXiv: 1302.7100] [INSPIRE].

[40] G. 't Hooft and M. Veltman, An illuminating discussion on causality in quantum field theory can be found, in Diagrammar, Section 6.1, CERN-73-09.

[41] N.N. Bogoliubov and D.V. Shirkov, Introduction to the theory of quantized fields, Interscience Publishers, New York, U.S.A., (1959).

[42] A.D. Linde, Inflationary Cosmology, Lect. Notes Phys. 738 (2008) 1 [arXiv:0705.0164] [INSPIRE]. 